\title{
La digitalización docente frente al reto de crisis sanitaria. La adaptación del claustro de Quirónsalud Madrid
}

\section{The digitalization of teachers in the SARS-CoV-2 pandemic challenge. The adaptation of the cloister of Quirónsalud Madrid}

\author{
Javier Bermejo', Carmen Rivero²; Sara Rojo ${ }^{3}$, Eva Cerezo ${ }^{4}$; Noemi Rodríguez ${ }^{5}$; M.Aranzazu \\ Carrasco $^{6}$; Jonatan Rodríguez $;$; M.Carmen Suárez ${ }^{8}$.
}

1 Responsable Asesoría Pedagógica Quirónsalud Madrid - Territorio 1; Universidad Europea de Madrid, Facultad de Ciencias Biomédicas; javier.bermejof@quironsalud.es, https:/ / orcid.org/0000-0003-3921-6346

2 Coordinadora Docencia e Investigación Complejo Hospitalario Ruber Juan Bravo; Universidad Europea de Madrid, Facultad de Ciencias Biomédicas; carmen.rivero@quironsalud.es, https://orcid.org/0000-0003$\underline{0249-6735}$

3 Coordinadora Docencia Hospital Universitario Quirónsalud Madrid; Universidad Europea de Madrid, Facultad de Ciencias Biomédicas. sara.rojo@quironsalud.es, https://orcid.org/0000-0002-1317-0530

4 Administrativa Territorial Docencia e Investigación; Universidad Europea de Madrid, Facultad de Ciencias Biomédicas. eva.cerezo@quironsalud.es, https:/ / orcid.org/0000-0002-7627-9410

5 Administrativa Territorial Docencia e Investigación; Universidad Europea de Madrid, Facultad de Ciencias Biomédicas. noemi.rodriguezbeas@quironsalud.es, https:/ / orcid.org/0000-0003-4517-7910

6 Asesora Pedagógica Complejo Hospitalario Ruber Juan Bravo; Universidad Europea de Madrid, Facultad de Ciencias Biomédicas. aranzazu.carrasco@quironsalud.es, http:/ / orcid.org/0000-0001-5760-8234

7 Asesor Pedagógico Hospital Universitario Quirónsalud Madrid; Universidad Europea de Madrid, Facultad de Ciencias Biomédicas. jonatan.rodriguez@quironsalud.es, https://orcid.org/0000-0003-4979-5126

8 Jefa Territorial Docencia e Investigación Quirónsalud Madrid - Territorio 1; Universidad Europea de Madrid, Facultad de Ciencias Biomédicas. carmen.suarezs@quironsalud.es, https://orcid.org/0000-0001$\underline{5594-3725}$

* Correspondencia: javier.bermejof@quironsalud.es

Recibido: 23/9/2020; Aceptado: 29/12/2020; Publicado: 4/1/2021

Resumen: La digitalización del profesorado universitario es un aspecto que ha cobrado especial relevancia en los últimos meses, de forma más concreta en el ámbito sanitario. Las clases presenciales se han visto canceladas y sustituidas por docencia online de una manera abrupta a causa de la crisis sanitaria acontecida. A ello se suma la elevada carga asistencial que han asumido todos los profesionales sanitarios. Varios de los hospitales del grupo Quirónsalud en Madrid desarrollan gran parte del plan académico del Grado de Medicina de la Universidad Europea y, debido a las circunstancias derivadas de la pandemia, su docencia ha tenido que transformarse, usando herramientas digitales que permitieran llevar a cabo tanto la labor docente como el arduo trabajo hospitalario de forma simultánea. Este artículo presenta los datos de la docencia llevada a cabo, así como las iniciativas que se han puesto en marcha desde marzo de 2020, con la finalidad de analizar la adaptación del claustro médico docente del grupo Quirónsalud a la situación acaecida. La utilización de herramientas asíncronas ha favorecido el trabajo autónomo del estudiante y la compatibilización de la actividad asistencial y la docencia en los hospitales mediante iniciativas como clases síncronas online, seminarios grabados y presentaciones locutadas, entre otras.

Palabras clave: Docencia médica; digitalización docente; COVID-19; TIC

Abstract: The digitalization of university teaching staff is an aspect that has taken on special
relevance in recent months, especially in the field of healthcare. Face-to-face classes have been
abruptly cancelled and replaced by online teaching due to the health crisis. In addition, all the
health care providers have taken on the burden of providing care, making it compatible with the
virtual classes. Several Quirónsalud groups hospitals carry out part of the academic plan for the 
degree in Medicine at the European University. However, the teaching has had to be transformed, using digital tools that allow the teaching work and the arduous hospital work to be blended. This article presents the data on the teaching carried out and the initiatives that have been implemented since March 2020 with the aim of analyzing the adaptation of the medical teaching staff of the Quirónsalud group to the situation that has occurred. The use of asynchronous tools has favored the autonomous work of the student and the compatibility of healthcare activity and teaching in hospitals through initiatives such as online synchronous classes, recorded seminars, and spoken presentations, among others.

Keywords: Medical teaching; teacher digitization; COVID; ICT

\section{Introducción}

Dadas las circunstancias particulares que presenta en la actualidad la docencia de la Medicina en los hospitales, ésta se encuentra en constante debate y transformación. Más allá de los avances didácticos y de recursos digitales para la docencia existentes hoy en día, la formación médica presenta diferentes características que hacen que los docentes que imparten clases teóricas y acompañan a los alumnos durante sus rotaciones, deban reconsiderar tanto su rol como su propia didáctica. Esta transformación surge de la necesidad de sustituir el rol del médico docente, tradicionalmente conceptualizado como transmisor de conocimiento especializado, al de facilitador del aprendizaje. La educación y el paradigma que la engloba son un proceso vivo, que se encuentran en continuo cambio, adaptándose a las demandas de la sociedad. Este rol se caracteriza por el dominio de, por un lado, la materia a impartir y por otro, la forma o modo de transmitir el conocimiento (1). El fundamento de esta premisa es lograr que el aprendizaje y la captación del conocimiento por parte del alumnado sea totalmente significativa, utilizando una metodología que fusione las características de la materia, las nuevas propuestas metodológicas innovadoras y la realidad educativa.

Dentro de esta necesidad de transformación es posible encontrar la famosa digitalización. En no pocas ocasiones, ésta se conceptualiza como la solución mágica a todo tipo de problemas. Sin embargo, resulta conveniente recalcar que toda herramienta digital, con independencia de su uso, no es la finalidad. En el ámbito educativo tampoco. Todo recurso digital debe considerarse como herramienta que apoye, facilite y mejore el proceso de enseñanza-aprendizaje (2). Con todo, conviene recalcar que según la Conferencia de Rectores de las Universidades Españolas (CRUE), éstas destacan por su capacidad de adaptación tecnológica.

En este sentido, resulta clave mencionar los organismos oficiales que desarrollan documentos y guías para que las entidades educativas puedan orientar este tipo de formación. Ejemplo de ello es la elaboración del Marco Común de Competencia Digital Docente que regula éstas, las cuales se componen de 5 áreas y 21 competencias (tabla 1), organizadas en 6 niveles, que permiten poner a prueba la capacitación del profesorado, de forma que se elaboren planes de formación acordes a las necesidades (3).

También existen otros estudios que buscan identificar las competencias con las que debe contar un profesor o profesora universitaria para integrar de una manera eficaz las TIC en su práctica diaria. Ejemplo de ello es el estudio de Rangel (4), que lleva a cabo una matriz de operacionalización, a través de la cual se extrajeron tres dimensiones que recogen las competencias necesarias: tecnológica, informacional y pedagógica. 
Tabla 1. Áreas y competencias del Marco Común de Competencia Digital Docente (3)

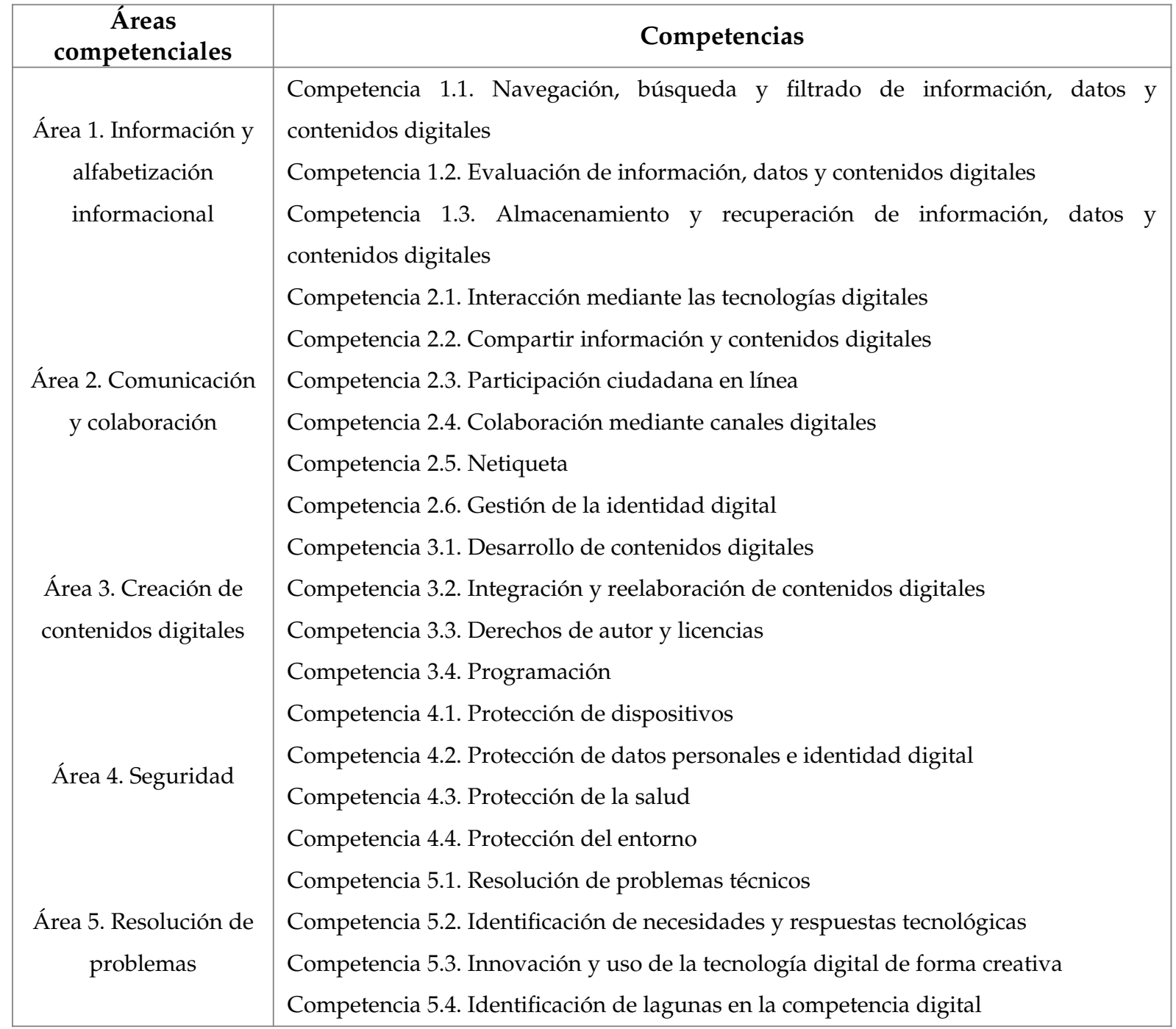

De igual forma, la utilización de las nuevas tecnologías permite llevar a cabo funciones distintas según las necesidades del entorno (5). Por ello, el propio profesorado universitario ve su uso como una herramienta positiva en el aula, que facilita el conocimiento científico (6) y que puede llegar a "romper las barreras espacio-temporales que han influido sobre las actividades formativas en los sistemas educativos universitarios convencionales" (5). Para el caso concreto de la docencia médica, el uso de herramientas digitales para el desarrollo de sus clases teóricas permite adecuar el proceso de enseñanzaaprendizaje a las diferentes eventualidades que pueden surgir.

Asimismo, para que el cambio al que aludíamos anteriormente se pueda llevar a cabo, se requiere una adecuación de la formación de los docentes al uso de las TIC y en esto tienen un papel decisivo las instituciones de educación superior que podrán gestionar el conocimiento y la innovación tecnológica (7). Para alcanzar tal fin, parece fundamental una correcta capacitación del docente en su uso, aspecto que son los propios docentes quienes demandan como esencial para su labor didáctica (6).

Desde el año 2008 el Hospital Quirónsalud Madrid se encuentra acreditado como hospital universitario y, a partir del 2013, se incorporaron a la docencia los hospitales Ruber Juan Bravo y Hospital Quirónsalud San José. Desde ese momento, la actividad docente de dichos hospitales se encuentra vinculada a la Universidad Europea en el Grado 
en Medicina, así como en otras actividades formativas y de investigación. Esta circunstancia hace que gran parte del cuadro médico sea también docente.

El pasado mes de marzo de 2020, a este contexto formativo y docente se planteó un doble reto. De un lado, el desafío de la digitalización masiva y forzosa por causas de la COVID-19, pues, dadas las circunstancias que acompañaron a la evolución de la pandemia, las universidades españolas, siguiendo las recomendaciones e indicaciones de las autoridades sanitarias, fueron comunicando el cierre de sus instalaciones, trasladando con ello la docencia al ámbito digital, fundamentalmente a través del formato de clases online. De otro lado, surgió el reto, nada desdeñable de que, el profesorado médico, que se encontraba en plena actividad asistencial por motivos de emergencia sanitaria, diese continuidad a su calendario docente en este nuevo formato.

Por todo ello, este artículo tiene el objetivo de analizar cómo ha sido la transición y adaptación del claustro docente de Quirónsalud Madrid a la docencia online durante la cuarentena. A partir de este objetivo general, se determinaron dos objetivos específicos:

- Identificar los principales recursos digitales utilizados por los profesores y profesoras durante el confinamiento.

- Estimar la adaptación de la docencia online al programa presencial planificado previamente.

\section{Métodos}

Hemos realizado un análisis observacional retrospectivo de las clases impartidas durante el estado de cuarentena en España en los hospitales del grupo Quirónsalud. Este análisis se llevó a cabo revisando las dinámicas realizadas durante los últimos 4 meses de docencia del curso 2019/2020, en los cuales, junto con la Universidad Europea se ha trabajado en la adaptación de las clases, los materiales y la metodología a la educación online. El objetivo de todo ello fue continuar con las materias y planificación educativa, sin perjudicar el correcto desarrollo del alumnado del Grado en Medicina, a pesar de la crisis sanitaria vivida.

El claustro docente, en lo que a la docencia teórica se refiere, se configura en base a 155 profesores asociados. La mayor parte de ellos se encuentra vinculado a una única asignatura y la actividad docente teórica se realiza de forma presencial en las aulas de los hospitales, utilizando el Campus Virtual propio de la Universidad para la notificación de información, inclusión de calendarios, gestión de actividades formativas y repositorio de materiales didácticos. De la totalidad del claustro detallado, en este estudio se cuenta con la muestra de los docentes que impartieron docencia durante el segundo semestre en los hospitales Quirónsalud Madrid (HUQM), Ruber Juan Bravo (CHRJB) y Quironsalud San José. Esta muestra se compone de 94 médicos, que se distribuyen en un 39.4\% de mujeres, y un $60.6 \%$ de hombres.

En lo que respecta a la estructura del Grado en Medicina en la Universidad Europea, éste se configura en seis cursos. Del total, los comprendidos entre cuarto y sexto se imparten de forma íntegra en los hospitales, es decir, tanto las clases teóricas como las prácticas de los estudiantes de Medicina, se realizan en las instalaciones de los mismos. Todas las asignaturas son semestrales a excepción de una en cuarto y dos en quinto que presentan carácter anual. Concretamente, las materias que se vieron afectadas por el confinamiento fueron:

- Formación clínica I - Aparato Respiratorio y Cardiología

- Formación clínica II - Digestivo y Hematología 
- Formación clínica III - Infecciosas

- Formación clínica IV - Nefrología y Urología

- Formación clínica V - Traumatología

- Formación clínica VI - Sistema Nervioso

- Formación clínica VII - Pediatría

- Formación clínica VIII - Dermatología

- Formación clínica IX - Ginecología

- Formación clínica X - Oftalmología

- Formación clínica XI - Otorrinolaringología

- Formación clínica XII - Geriatría

Para la recolección y análisis de datos se elaboró un protocolo de recogida y registro de la información durante los meses de cuarentena, así como el seguimiento de las aulas virtuales por parte de la Asesoría Pedagógica del centro. Para este registro se utilizó el programa Excel del paquete Office, lo que permitía a las personas implicadas en la recolección de datos realizarlo de forma colaborativa en remoto.

\section{Resultados}

En la Figura 1 se puede ver la distribución de docentes según la asignatura y hospital en el que imparten docencia. Se puede observar que hay una distribución homogénea entre hospitales, excepto en las últimas cuatro asignaturas, ya que son dos Formaciones Clínicas que se imparten en semestres alternos en los hospitales, por lo tanto, solo tenemos muestra de uno de ellos. Observamos que hay un promedio de 4 profesores en cada asignatura, con un mínimo de 1 profesor en la asignatura de Geriatría de CHRJB y un máximo de 8 en Sistema Nervioso y Digestivo de CHRJB. Desde marzo de 2020 hasta la finalización del semestre se han registrado 106 horas de docencia virtual de forma síncrona a través de BlackBoard Collaborate y se han locutado 284 horas de presentaciones que, de forma asíncrona, los estudiantes pudieron visualizar. En relación a las clases síncronas destaca especialmente la asistencia del alumnado a las mismas, siendo muy superior a la asistencia presencial durante el primer semestre.

Previo a este estudio, durante el curso académico 2019-2020, se realizó un análisis sobre la digitalización del claustro, con el horizonte de identificar el punto de partida del mismo. En dicho análisis se identificó que tan solo el 4,96\% del claustro, durante sus clases teóricas, usaba alguna herramienta digital con carácter educativo que no fuese el tradicional PowerPoint de presentación. Adicionalmente, se observó que un 6,83\% del total de docentes, realizaba alguna metodología innovadora alejada de la clase magistral (apoyada o no en recursos digitales).

Fruto de este estudio se realizó un análisis de las metodologías utilizadas por parte del claustro docente durante el confinamiento y se detectó que el $86,67 \%$ de las Formaciones Clínicas impartidas, y el 41,5\% del claustro docente utilizaron Herramientas Pedagógicas Digitales (HPD), incluyendo las clases síncronas. En la Figura 2 se puede observar la distribución de ese uso entre el numero total de docentes de cada Formación Clínica que si utilizó este tipo de herramientas durante el confinamiento.

De entre los docentes que usaron HPD durante el periodo de estudio, se pueden diferenciar varias herramientas, como son los videos explicativos, uso de gamificaciones y clases interactivas en directo. Concretamente, el $20 \%$ utilizaron los videos explicativos como herramienta, en los que el profesorado explica a través de una grabación aspectos más prácticos de la asignatura, como, por ejemplo, la realización de suturas o una punción lumbar. 


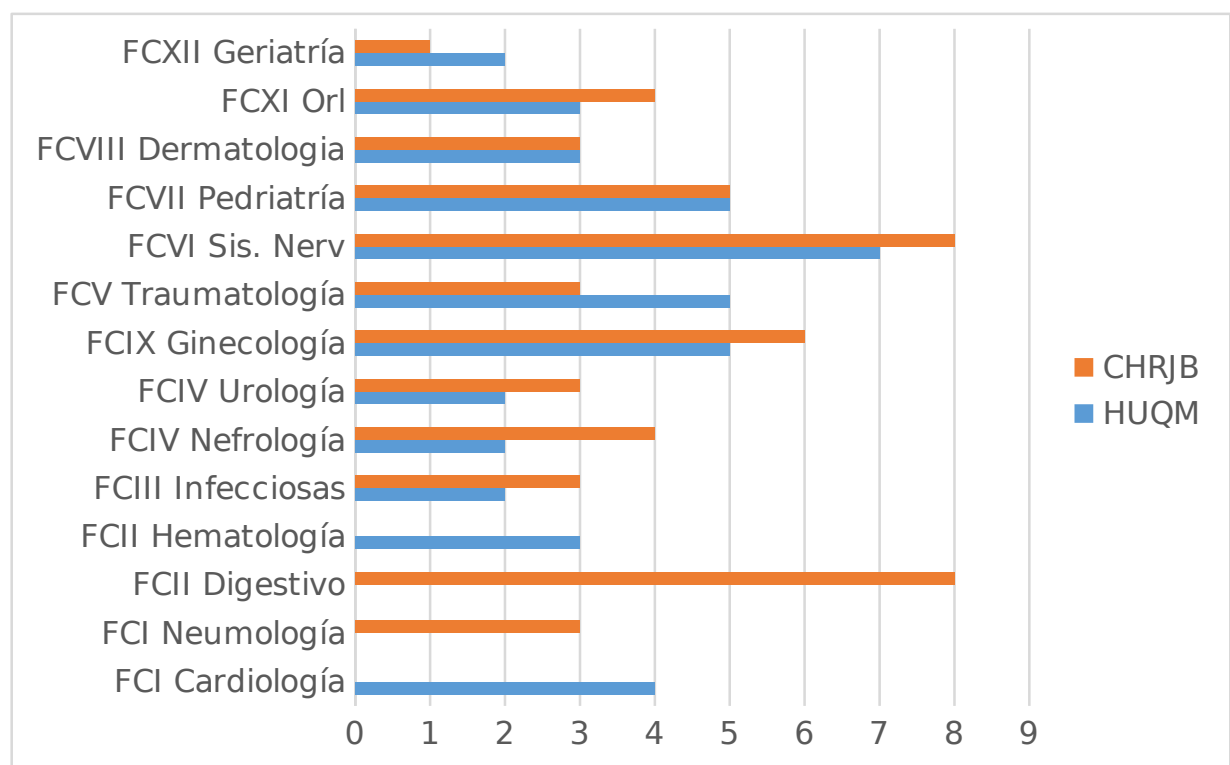

Figura 1. Distribución de docentes por Formación Clínica y Hospital.

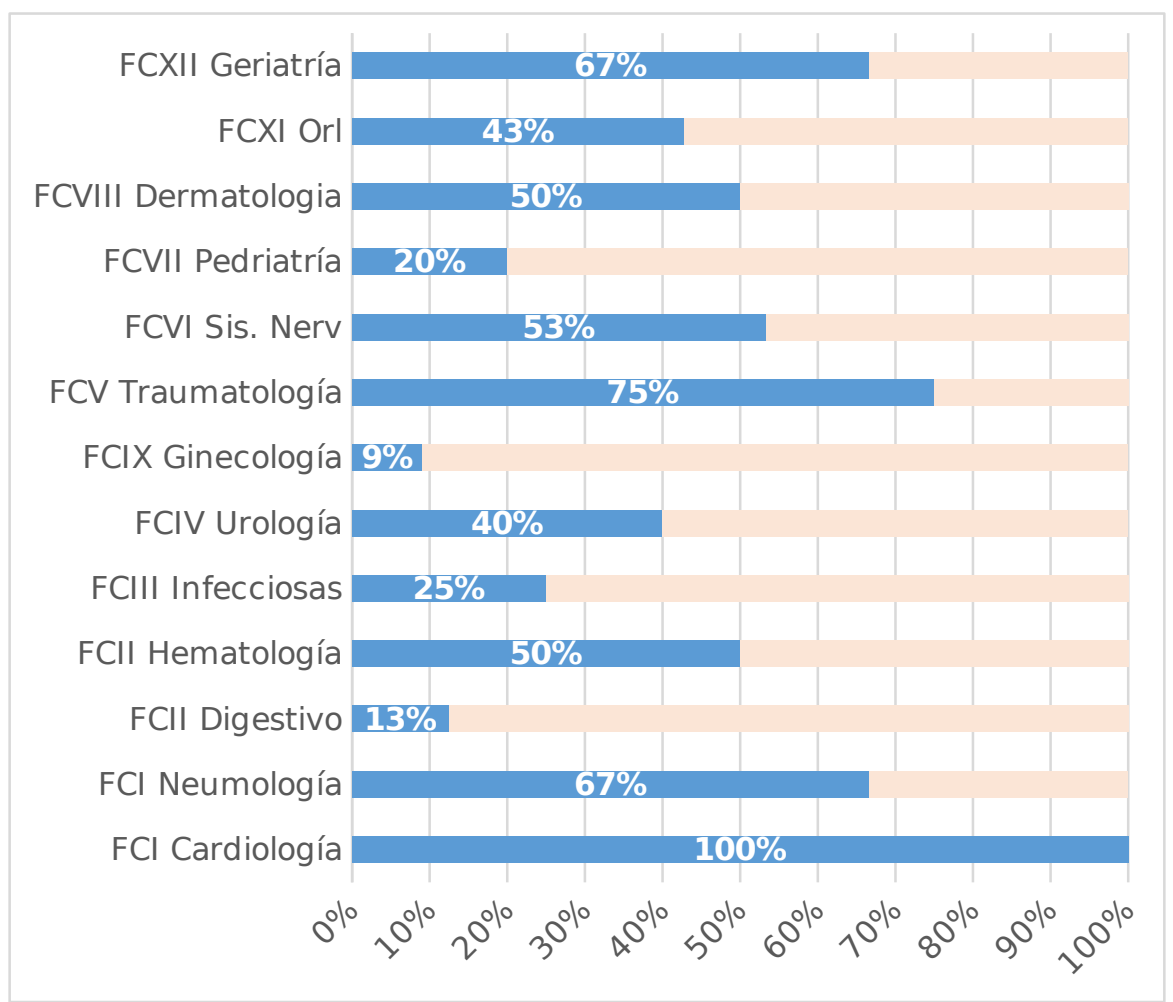

Figura 2. Uso de Herramientas Pedagógicas Digitales según Formación Clínica.

El 7.7\% del profesorado que usó HPD realizó docencia utilizando la aplicación Socrative integrada en los talleres interactivos, lo que permitía al alumnado ir autoevaluándose y al docente adaptar la explicación a las necesidades del estudiante. Finalmente, el $74.4 \%$ utilizó las clases síncronas e interactivas para impartir la docencia. Estas clases se llevaron a cabo a través del Aula Virtual del Grado de Medicina.

Por otro lado, el $80 \%$ de las asignaturas impartidas utilizaron presentaciones locutadas, la entrega de documentos y casos clínicos con preguntas a responder por los 
estudiantes o el uso de videos ya subidos a plataformas como Youtube o Vimeo. Concretamente, una de las herramientas más extendidas fueron las Presentaciones Locutadas, que en la Figura 3 podemos observar que se utilizaron por el $80 \%$ de las Formaciones Clínicas y que su uso medio fue del $68,5 \%$

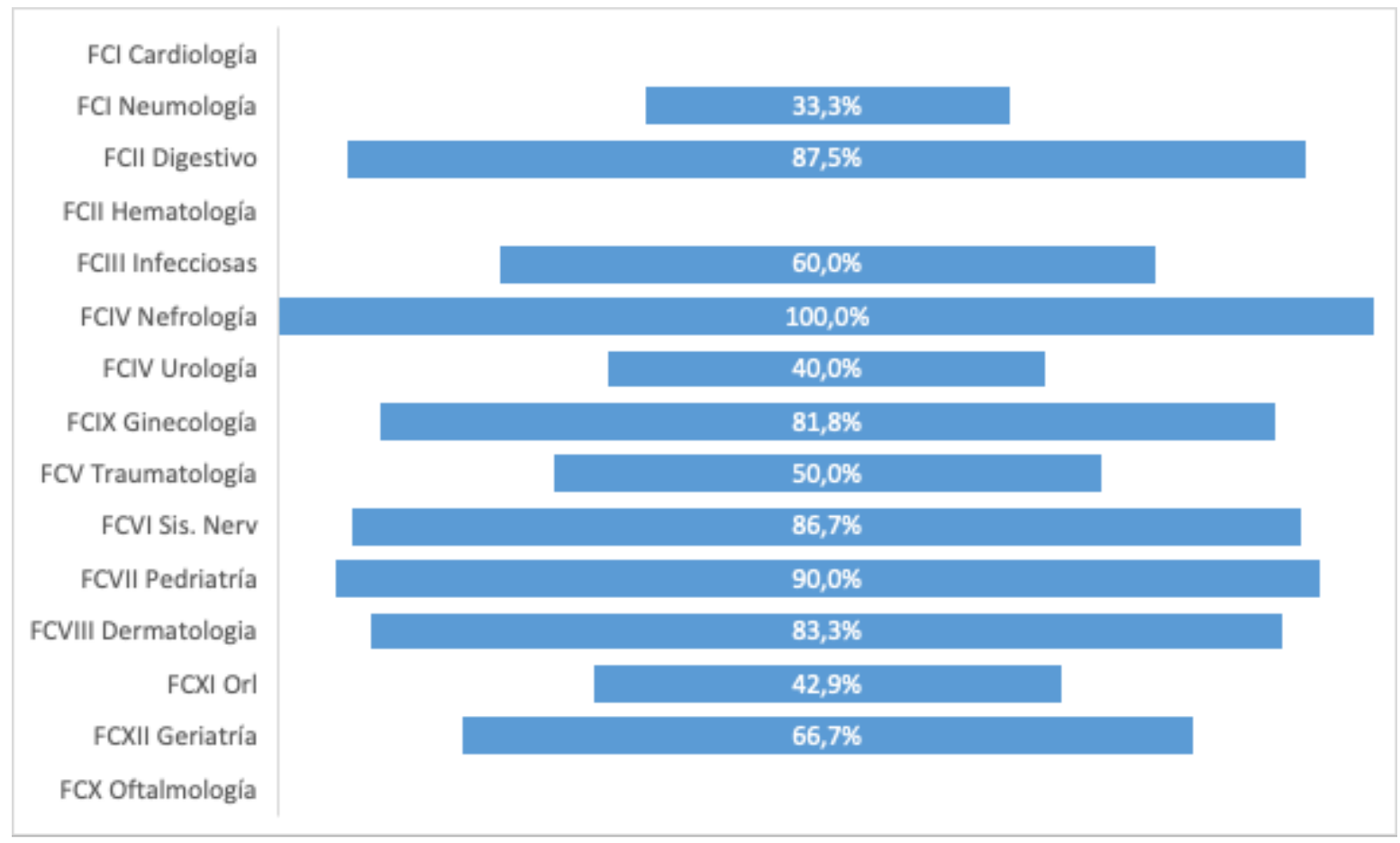

Figura 3. Uso de Presentaciones Locutadas según Formación Clínica.

Por último, hay que indicar que casi el $40 \%$ de los docentes evaluados utilizaron más de una herramienta a lo largo del cuatrimestre, entre las HPD, videos ya subidos, entrega de casos clínicos y presentaciones locutadas. Además, se intensificó el uso del Aula Virtual para compartir materiales con los estudiantes.

De otra parte, la evaluación de las materias se llevó a cabo a través de pruebas que midieran el conocimiento del alumnado de manera objetiva. Para poder llevarlo a cabo, se optó por pruebas online tipo test. En este sentido, destacar que la Universidad Europea dotó de un sistema de e-proctoring a la plataforma. Se entiende por e-proctoring a aquel sistema que permite la vigilancia de vídeo y audio utilizando los medios técnicos del estudiante, con el objetivo de prevenir cualquier práctica deshonesta de copia en la prueba de evaluación (8). Debido al cambio de paradigma de la educación en este periodo, resulta necesario tomar a la tecnología como una de las aliadas más importantes. Los sistemas e-proctoring ayudan a evitar el fraude y las prácticas deshonestas por parte del alumnado, consiguiendo así que las pruebas sean objetivas y que midan el conocimiento real que tiene el examinado.

Por último, respecto a los resultados logrados con el cambio de paradigma, destacar que éstos no se han visto afectados significativamente. Este dato demuestra que los alumnos se han adaptado adecuadamente a la modificación metodológica realizada. Por tanto, en caso de posibles emergencias sanitarias u de otra índole, esta nueva modalidad formativa, podrá ser considerada para desarrollarse. En definitiva, la pandemia ha modificado la visión de la formación a gran escala, posicionando la formación online como metodología real de cambio en la realidad educativa. 


\section{Discusión}

Como ya ha sido indicado, la digitalización de los docentes es un hecho que en la actualidad ha adquirido una especial relevancia por la situación tan excepcional vivida a nivel mundial. Esto ha provocado un cambio radical en la metodología docente existente hasta el momento, poniendo de manifiesto la necesidad de profundizar en la capacitación del profesorado en herramientas tecnológicas acordes a las nuevas necesidades. Si la digitalización docente es un reto que acompaña a la actividad del profesorado durante las últimas décadas, a tenor de las circunstancias derivadas acontecidas en la actualidad, se ha hecho todavía más evidente. Con todo, es sabido que la digitalización no es un asunto novedoso, pues se encuentra abundante literatura pedagógica sobre el tema, siendo un ámbito de investigación ampliamente trabajado.

El claustro docente de Quirónsalud en Madrid, parte de una escasa digitalización educativa y un uso generalizado de la clase magistral, tal y como se detecta en el estudio preliminar elaborado, donde menos del 5\% de docentes utiliza alguna herramienta digital, más allá del Power Point. En la actualidad se hace imperante la necesidad de tener control sobre las tecnologías existentes, ya que, sin ello, será mucho más difícil superar las barreras que nos encontramos en su uso (9). Una de las principales funciones del docente digital es la de apoyar el desarrollo de la competencia "aprender a aprender" en los estudiantes y que éstos se encuentren integrados en la cultura del cambio, así como la promoción del pensamiento crítico apoyándose en las herramientas de información que tienen a su alrededor (4). Para ello, es necesario que el propio docente digital conozca esas herramientas de información que mencionamos, que actualmente vienen acompañadas y se encuentran integradas en las TIC.

Hoy en día, la herramienta más extendida como soporte para la formación e-learning, es el Aula Virtual. Como es sabido, estos entornos ya no se conciben como herramienta que se utilice únicamente para los modelos virtuales de docencia (10), sino que se han convertido en una herramienta más a incluir en las programaciones didácticas de los grados presenciales, ya que ofrece multitud de opciones formativas que enriquecen el proceso de enseñanza-aprendizaje. Esta cuestión se ha visto especialmente activada en los últimos meses, en los cuales, los docentes de todo el mundo han tenido que adaptar su docencia presencial de forma forzosa a estas herramientas y cambiar el paradigma de la educación presencial. En el caso de la rama sanitaria, esta transformación ha sido especialmente compleja, a causa de la actividad asistencial, ya que, la carga de trabajo ha aumentado de manera significativa con el colapso del sistema sanitario.

Actualmente, existen multitud de proveedores tecnológicos de Aulas Virtuales con funcionalidades diversas. En el caso particular que nos ocupa, el Grado en Medicina de la Universidad Europea y de forma más concreta, en los hospitales del Grupo Quirónsalud en Madrid, el Aula Virtual utilizada fue BlackBoard Collaborate (integrada en el Campus Virtual de BlackBoard). En ella, los médicos docentes han impartido sus clases en vivo (sesiones síncronas e interactivas) que han sido grabadas para, posteriormente, poder ser publicadas en la plataforma, facilitando así la visualización y acceso a los contenidos didácticos por parte de todo el alumnado. En este sentido, resulta conveniente destacar dos premisas. La primera es que, dadas las circunstancias sanitarias y de confinamiento, podría sobre entenderse que la asistencia, por parte de los estudiantes, a este tipo de clases online síncronas sería masiva. Sin embargo, al mismo tiempo, el hecho de que los alumnos pudieran disponer de estas clases grabadas para poder ser visualizadas en cualquier momento, suponía un escenario desconocido para el claustro docente del Quirónsalud 
Madrid. En este sentido, los porcentajes de asistencia se vieron ciertamente incrementados con respecto a los datos de asistencia presencial.

Los recursos utilizados en esta plataforma fueron diversos y adaptables a cada objeto de aprendizaje. En las clases, además de su impartición síncrona y grabación, se posibilitó la opción de que los docentes pudieran locutar sus presentaciones de forma asíncrona, en formato PowerPoint. Dichas presentaciones eran puestas a disposición de los alumnos como material complementario a las sesiones en directo o en su defecto como material directo de estudio. Este recurso sirve al alumno, como material de seguimiento durante la preparación de la asignatura, con el aliciente de disponer de las explicaciones del docente en formato audio integrado en un único archivo. A pesar de que el principal riesgo que presupone la formación online es la pérdida de contacto directo con el docente, en el caso concreto de Quirónsalud, se puso a disposición del alumnado no sólo el chat online accesible en el Aula Virtual o la posibilidad de interacción por micrófono, sino el contacto directo para la resolución de dudas vía email. Este formato también fue utilizado para la impartición de seminarios. Si bien estos, en algunos casos tenían un objeto más dinámico, orientado hacia la interacción, los docentes utilizaron diferentes recursos para desarrollarlos. Aplicaciones como Socrative o Kahoot, fueron las más recurrentes.

Sin embargo, hemos de tener presente que el uso de las tecnologías en el aula conlleva una serie de desventajas que se deben controlar adecuadamente. En el estudio de Ferro et al. (5) se detectan, entre otras, "el considerable aumento de informaciones no fiables; las dificultades para localizar, seleccionar y analizar la información; los riesgos de ansiedad o adicción en los estudiantes por la continua interacción con el ordenador; los problemas de falta de sociabilidad o la aparición de dolencias (cansancio visual y otros problemas físicos provocados por malas posturas ocasionadas por un exceso de tiempo trabajando ante el ordenador)".

De forma adicional, existen otro tipo de barreras más orientadas a las características del docente, como pudieran ser el desconocimiento o resistencia al cambio, que, además, en la situación acontecida en los últimos meses, ha podido ir acompañado de una frustración ante la combinación de la imperiosa necesidad de cambio y dicha resistencia. Es por ello, por lo que resulta necesario desarrollar una formación continua en digitalización docente que permita tener un reciclaje continuo de todas nuestras competencias tecnológicas (7). Sin embargo, regresando a la situación producida por la COVID-19, la extrema complejidad existente para facilitar la continuidad del curso académico en general (11), se ha visto incrementada más si cabe, en el caso de la docencia sanitaria, la cual ha tenido una especial carga asistencial.

Bien es cierto que la pandemia ha tenido repercusión en todos los ámbitos de la Sociedad, pero especialmente, se ha observado una importante crisis en Sanidad, Economía o Educación. En cuanto a la Economía la COVID-19, ha provocado grandes daños a nivel mundial. La ruptura de las cadenas de producción, distribución y consumos han provocado pérdidas incalculables para cada país (12). Las medidas extraordinarias tomadas por los gobiernos, como el confinamiento de la población o la paralización de las actividades del país, han interferido en todas las estructuras sociales a nivel mundial. Del mismo modo, esta situación ha afectado a la Educación ya que, ha creado una brecha digital de acceso a la formación en aquellas familias con un nivel socioeconómico inferior. La brecha de acceso, es definida por García-Peñalvo (13) como la falta de "acceso a la tecnología, a los ordenadores, a los dispositivos móviles de altas prestaciones y, principalmente, a una adecuada conexión a Internet. Se distinguen dos situaciones, principalmente: la falta de acceso por carecer de medios económicos y la falta de acceso por residir en zonas con problemas de despliegue de las redes de comunicación". Esta 
brecha aparece debido a la necesidad de incorporar dispositivos tecnológicos (tablets, dispositivos móviles, PCs, etc.) y recursos como Internet en familias que no disponen de recursos para poder adquirirlos.

Por otro lado, la Educación no ha sido ajena a este acontecimiento. Según datos recabados por UNESCO, la pandemia es la responsable de la suspensión de las clases de más de 1.570 millones de estudiantes de cualquier nivel educativo en todo el mundo (14). A finales del mes de marzo de 2020 fueron más de 166 países los que habían cerrado las aulas, afectando a más del $87 \%$ de la población estudiantil a nivel global, aumentando a 192 países en abril y al 91,4\% de la población estudiantil mundial $(12,15)$. En nuestro país son alrededor de 10 millones de estudiantes los que se han encontrado, prácticamente, de un día a otro viendo trasformado su sistema educativo, y por consiguiente el cambio de metodologías docentes de una forma atropellada. Estos datos han supuesto la adaptación del Sistema a la realidad educativa que está viviendo la sociedad. Las plataformas de formación online, webinars y sesiones locutadas aparecieron para intentar dar respuesta a la necesidad de formación que demandaban los estudiantes. La formación online surge como metodología de ayuda a la vuelta a la normalidad de toda la educación.

Independientemente de todo ello, el ámbito universitario lleva años abordando la digitalización como propuesta de mejora en las aulas, sin embargo, para poder llevar a cabo un proceso de digitalización resulta necesario modificar más aspectos que los contenidos o los procesos (16). La metodología utilizada en la educación online, debido a los medios utilizados y a las particularidades, presenta características que ayudan a diferenciarse de la utilizada en la educación presencial, pudiendo incluso complementarla. Los contenidos de la formación online no deberían ser copias de la formación presencial. Por ello requieren que, tanto la estructura como los propios contenidos a explicar, sean adaptados tanto al entorno digital en el que se desarrollan, como a la metodología de enseñanza y aprendizaje que posibilita el propio escenario digital. La situación del aprendizaje debería ser lo primero en la mente de las personas que gestionan e imparte formación online (17). Asimismo, una de las principales ventajas que presenta la formación online es la posibilidad de disponer de varias cuotas de sincronía (chats) y presencialidad (clases en directo) (18).

En situaciones normales el paso de la educación presencial a educación online o no presencial resulta complejo, debido a las adaptaciones pedagógicas que son necesarias realizar. Sin embargo, la situación de la COVID - 19 responde a razones de emergencia y excepcionalidad que provocan que los cambios sean de la misma naturaleza. La realidad vivida ha marcado los ritmos de adaptación de la Educación, obligando a la transformación radical de la formación presencial a formación online. Este paso se realizó sin la planificación oportuna debido a la situación que se estaba viviendo, ya que, no se puede pretender que esta experiencia hubiera sido similar que de haberlo hecho de manera planificada (19).

\section{Conclusiones}

- La pandemia vivida ha puesto de manifiesto diferentes necesidades a nivel global. En el ámbito educativo, qué duda cabe, la necesaria adaptación a la digitalización ha encontrado barreras, problemas, pero también ha permitido identificar algunas cuestiones de interés para el futuro. Más allá de los pormenores de la adaptación forzada, así como de los conflictos y dificultades derivadas de la misma, parece que el reto de la docencia online ha llegado para quedarse. Esto no implica que la formación universitaria cambie radicalmente su perspectiva de lo presencial a lo virtual, pero con 
cierta probabilidad, muchos docentes habrán encontrado en las TIC, diferentes recursos que, a pesar de que se supere la situación sanitaria actual, utilizarán en el futuro.

- De otro lado, como ha sido detallado en los epígrafes anteriores, apostar por la digitalización no supone fomentar el uso de recursos digitales, sino más bien, favorecer la formación de los docentes en el uso de herramientas de carácter digital. Ese cambio hacia la digitalización, tan promulgado desde muchas esferas, requiere de una apuesta verdadera no sólo por parte de los docentes, sino de las propias instituciones educativas. Este nuevo mundo digital que se ha abierto ante nosotros, lo ha hecho, también, para el alumnado, quien ha encontrado un ámbito a explorar y que explotar al mismo tiempo. La innovación tecnológica asumida por los docentes en los últimos meses ha supuesto un gran cambio en materia formativa. Este desarrollo ha proporcionado acceso a los estudiantes a nuevas experiencias formativas, expresivas y educativas, permitiendo así actividades difícilmente imaginables en el aula. Tal vez por ello, la modalidad de educación online se encuentra cada vez más demandada, pues los beneficios que presenta permiten romper las barreras-espacio-temporales en todos los sentidos, flexibilizando el proceso de enseñanza-aprendizaje (5).

- Otra de las conclusiones a destacar, sino la más importante, es la capacidad de respuesta del personal sanitario. Más allá de las dificultades vividas, han demostrado un compromiso ímprobo con la educación. El claustro de Quirónsalud Madrid ha colaborado de forma activa en la continuidad del programa formativo en la Universidad Europea, no solo adaptándose a los requisitos estipulados sino ofreciendo, en muchas ocasiones, soluciones innovadoras de carácter pedagógico.

- Con todo, la oportunidad fundamental que, a nivel educativo, ha emergido con la COVID-19, es la necesidad de que las instituciones educativas formen a sus docentes en herramientas digitales, con el fin de que, más allá de anteponerse a posibles situaciones excepcionales, puedan superar los prejuicios y resistencia al cambio, ofreciéndoles recursos extensivos a sus clases tradicionales. Si el rol del médico docente se encuentra actualmente en cambio, por las demandas tanto formativas como por la evolución propia del ámbito médico, la digitalización docente no puede permanecer al margen.

Declaración de conflicto of interés: Los autores declaran no tener ningún conflicto de intereses

\section{Referencias}

1. Bermejo Fernández-Nieto J, Suarez Sánchez MC. La función pedagógica del médico docente como oportunidad. Anamnesis y tratamiento. Educ Med, 2019, In press, DOI: https://doi.org/10.1016/j.edumed.2019.10.017.

2. Selwyn, N. Profesores y tecnología. Repensar la digitalización de la labor docente. Boletín de la Institución Libre de Enseñanza, 2016, 104, 27-36.

3. INTEF. Marco Común de Competencia Digital Docente. Aprende Intef [Internet] 2017 [acceso 24 de junio de 2020], 1-83. Disponible en: https://aprende.intef.es/sites/default/files/2018-05/2017_1020_MarcoComún-de-Competencia-Digital-Docente.pdf

4. Rangel A. Competencias Docentes Digitales: Propuesta de un perfil. Rev de Medios y Educación, 2015, 46, 235-248. DOI: http://dx.doi.org/10.12795/pixelbit.2015.i46.15

5. Ferro, C, Martínez, AI, Otero, MC. Ventajas del uso de las TIC en el proceso de enseñanza-aprendizaje desde la óptica de los docentes universitarios españoles. EDUTEC, Rev Electrónica de Tecnología Educativa, 2009, 29, 1-12. DOI: https:/ / doi.org/10.21556/edutec.2009.29.451

6. Montoya, NE, González Palacio, EV. Competencias TIC en docentes de nivel técnico y tecnológico. Un estudio de caso en un centro de formación del SENA. Revista Virtual Universidad Católica del Norte, 2019, (58), 74-95. DOI: https:/ / doi.org/10.35575/rvucn.n58a3 
7. Ocaña-Fernández, Y, Valenzuela-Fernández, L, Morillo-Flores, J. La competencia digital en el docente universitario. Propósitos y Representaciones, 2020, 8(1), e455. DOI: http://dx.doi.org/10.20511/pyr2020.v8n1.455

8. García-Peñalvo, F, Corell, A, Abella-García, V. Grande, M. La evaluación online en la educación superior en tiempos de la COVID - 19. Education in the knowledge society (EKS), 2020, 21(4), 1-26. DOI: https://doi.org/10.14201/eks.23013

9. Ottenbreit, A, Liao, J, Sadik, O, Ertmer, P. Evolution of Teachers' Technology Integration Knowledge, Beliefs, and Practices: How Can We Support Beginning Teachers Use of Technology? Journal of Research on Technology in Education, 2018, 50(4), 1-23. DOI: https://doi.org/10.1080/15391523.2018.1487350

10. Gross Salvat, B. La evolución del e-learning: del Aula Virtual a la red. Rev Iberoamericana de Educación a Distancia, 2018, 21(2), 69-82. DOI: http:/ / dx.doi.org/10.5944/ried.21.2.20577

11. Almazán Gómez, A. Covid-19: ¿Punto sin retorno de la Digitalización de la Educación? Revista Internacional de Educación para la Justicia Social, 2020, 9(3e), 1-4. Recuperado a partir de https://revistas.uam.es/riejs/article/view/12089

12. Alcántara, A. Educación superior y COVID - 19: Una perspectiva comparada. En: Girón, J, Educación y pandemia: Una visión académica. México: Instituto de Investigaciones sobre la Universidad y la Educación (IISUE); 2020. 75 - 82.

13. García-Peñalvo, F. El sistema universitario ante la COVID - 19: corto, medio y largo plazo. Universidadsi [Entrada en blog] 2020 [acceso el 25 de junio de 2020]. Disponible en: https://www.universidadsi.es/sistema-universitario-covid-19/

14. UNESCO. El coronavirus COVID - 19 y la educación superior: impacto y recomendaciones. IESALC [Internet] 2020 [acceso el 25 de junio de 2020]. Disponible en: http:/ / www.iesalc.unesco.org/2020/04/02/el-coronavirus-covid-19-y-la-educacion-superior-impacto-yrecomendaciones/

15. Martínez, P. y Díez, F. La crisis del coronavirus afecta a 1.570 millones de estudiantes: ¿cómo encarar el tsunami? The conversation [Internet] 2020 [acceso el 25 de junio de 2020]. Disponible en: https://theconversation.com/la-crisis-del-coronavirus-afecta-a-1-570-millones-de-estudiantes-comoencarar-el-tsunami-136408.

16. García-Peñalvo, FJ. Modelo de referencia para la enseñanza no presencial en universidades presenciales. $\begin{array}{lllll}\text { Campus } \quad \text { Virtuales, } & \text { 2020, } & \text { E(1), }\end{array}$ http:/ / uajournals.com/ojs/index.php/campusvirtuales/article/view/625

17. Rodenes, M, Salvador, R, Moncaleano G. E-learning: características y evaluación. Ensayos de economía, 2013, (43), 143-159. En https:/ / revistas.unal.edu.co/index.php/ede/article/view/42932

18. Grau-Perejoan, O. Formación on-line. Educación Médica, 2008, 11(3), 139-146. En http:/ / scielo.isciii.es / scielo.php?script=sci_abstract\&pid=S1575-18132008000300006

19. Hodges, C, Moore, S, Lockee. B, Torrey, T, Bond, A. The difference between emergency remote teaching and online learning. Educase review [Blog] 2020 [acceso el 25 de junio de 2020]. Disponible en: https:/ / er.educause.edu/articles/2020/3/the-difference-between-emergency-remote-teaching-andonline-learning.1.

(C) 2021 por los autores. Enviado para su publicación en acceso abierto bajo los términos y condiciones de la licencia Creative Commons Attribution (CC BY) (http://creativecommons.org/licenses/by/4.0/). 